\title{
K-means algorithm with level set for brain tumor segmentation
}

\author{
Samah Abdelaziz ${ }^{1}$, Songfeng $\mathbf{L u}^{2}$ \\ ${ }^{1,2}$ School of computer Science and Technology, Huazhong University of Science and Technology, China \\ ${ }^{2}$ Shenzhen Huazhong University of Science and Technology Research Institute, China \\ ${ }^{1}$ Faculty of science, Zagazig University, Egypt \\ ${ }^{2}$ Nanjing Souwen Information Technology Co., Ltd., China
}

\begin{tabular}{l}
\hline \hline Article Info \\
\hline Article history: \\
Received Oct 1, 2018 \\
Revised Dec 10, 2018 \\
Accepted Jan 25, 2019 \\
\hline
\end{tabular}

\section{Keywords:}

Active contour model

Brain tumor MRI

Image segmentation

Level set method

\begin{abstract}
Brain is a complicated structure consisting of millions of millions cells so that, it's difficult to identify any diseases without using any computerized technology. Magnetic Resonance Imaging (MRI) is one of the main assessments of brain tumors. One of the most important steps on medical image processing is segmentation. Segmenting brain MRI images, which provide accurate information for the diagnosis and therapy decisions of brain tumors. We proposed to segment brain tumor MRI images into three parts (WM (white matter), GM (gray matter), and background). The first algorithm is for applying median filtering on brain MRI image for removing the noise from the image for achieving accurate results. The second algorithm is for applying K-means algorithm for accuracy in time consuming and for clustering into regions and the third algorithm indicate the detecting the boundary of the image with the use of level set. By comparison, our proposed method, its efficiency to segment perfectly more than other previous used algorithms especially on time consuming.
\end{abstract}

Copyright () 2019 Institute of Advanced Engineering and Science. All rights reserved.

\section{Corresponding Author:}

Songfeng Lu,

School of computer Science and Technology,

Huazhong University of Science and Technology, Wuhan 430074, China.

Email: lusongfeng@hust.edu.cn

\section{INTRODUCTION}

Image segmentation is an important process generally in image processing and especially in medical image processing $[1,2]$. It's still open area for many researches. There are numerous studies and a lot of challenges on image segmentations to obtain on an accurate method for an efficient segmentation process. By dividing image into different regions and parts in which each region has a group of pixels with a similar characteristics such as intensity, texture, or color [3]. There are many application can be applied in analysis, object recognition, object-based image compression and classifications processes [4, 5].

In segmentation, there are a lot of algorithms are used such as edge detection, region-based, clustering, level set and histogram based [6]. It is aiming to identifying, locating, characterizing, detecting and extracting a set of regions or contours of the image [7].

In medical image processing, image segmentation is a way to determine the region of tumors and sometimes tends to compute the volume of tumors $[8,9]$. According to the National Cancer Institute Statistics (NCIS) The National Brain Tumor Foundation (NBTF) for research in United States estimates that brain cancer percentage is increasing every year in U.S and UK and reported that it is the cause of increasing the percentage of death and also in India a huge number of people are affected by different types of tumors [10]. Brain is a complicated structure consisting of millions of millions cells so that, it's difficult to identifying any diseases without using any computerized technology [11]. According to medical experts, diagnosis process will be a time consuming process and there will be a life -threatening. So, image processing with medicine field is a great achievement. 
Brain tumor images segmentation is not easy process that using for identifying features of the normal and abnormal regions and separating them. These regions with tumors can be identified by increasing the size of the tissue, changing in shapes of the normal structure or appearing new extra cells [12]. So, we proposed to segment brain tissue images by separating and extracting white matter from gray matter.

We used MRI on our work as imaging modality for capturing the interior structure of the brain. This computerized technique is capable of imaging in variant directions and also quite safe more than other techniques as CT (computed tomography) and X-Ray [12].

There are several segmentation methods that applied on medical image for extracting, detecting, identifying, or separating tumors regions from healthy one. Several techniques are used in segmentation for brain MRI [13] but, the best methods that are used are clustering for Magnetic Resonance (MR) brain tumors where the number of clusters is usually well known for regions of interest in the image [14].

K-means algorithm is an important algorithm used in Medical image processing. After comparing between three segmentation techniques based on K-means, K-Mediods, and Hierarchical clustering techniques The authors in [15] deduced that the best technique in performance and easier to implement than the other techniques is K-means Clustering. However, the efficiency of K-means for helping in the process of segmentation without time consuming, it's critical with noises that can affect on the accuracy of results. Many researchers do all their bests to improve the accuracy of results by removing noises from brain MRI images which contain noises such as linear filters or non-linear filters, for example, the authors in [16] proposed to reduce the noise on the image first by using wavelet transform and then applying K-means clustering algorithm. Clustering algorithms have many applications in medical field as image segmentation.

We used median filter where it is one of the most important non-linear filtering for removing or reducing the noise on the images. It's an efficient method that can preserve the edge features and numerous of studies confirm that median filter is better than linear filtering for removing the noise on the processed image [17]. We introduced an efficient clustering algorithm that has ability to detect and identify the affected part in brain MR based on level set methods. Level set method was first mentioned by Sethian and Osher in 1988 [18]. We discussed the level set on section 4.

We introduced an efficient algorithm for brain MRI segmentation by developing Median filter for making the image a clear without noises with clustering images by using K-means clustering by showing the initial and final clusters with level set segmentation and after segmentation we are able to extract gray matter from white matter.

This paper describes the research as follows, Section 2 discuss related works. Section 3 discusses proposed method. Section4 describes segmentation with level set method. Section 5 discusses experimental results, followed by a conclusion in Section6.

\section{RELATED WORK}

following [19]:

There are most of problems that face numerous studies and have a great challenges for solving it as

a) Lack of experience for identifying the objects.

b) Lack of image quality.

c) Lack of techniques quality that can be used for capturing the image.

d) Detecting the image and localizing the position of the pathology.

e) Difficulties that face the most of researches of being the irregular part on the image inside or on the boundary of the image.

f) Luminance quality and environment quality that around the patient and image devices.

Researchers who are working on brain MRI images segmentation are using different approach for solving the problems that face the numerous studies. There are many algorithms for medical image segmentation

Abd-Ellah et al, [20] proposed to improve the accuracy of the design and the system of diagnosis without time consuming. They proposed also to classify brain tumor MRI images to normal and abnormal and then classify the type of the tumor to benign (non-cancerous) and malignant (cancerous). They used Kmeans clustering for segmentation, and discrete wavelet transform (DWT) for feature extraction by transforming images from spatial domain to frequency domain by using high pass and low pass filters on the brain tumor MRI images, and feature reduction by applying principle component analysis (PCA). Classification by using support vector machine (SVM).Koley et al, [21] proposed to determine the exact location of brain MRI image by using Cohesion based merging based on K-means clustering algorithm for partitioning the brain MRI image to identify the exact location of the tumor. K-means clustering used for clustering the image into different regions and each region has similarities characteristics and other regions with different characteristics, by merging all similarities region and then they apply CSM algorithm for 
calculating the inter cluster similarity for the regions with different characteristics to identify the region of the tumor. And finally to extract the tumor they applied erosion algorithm which is a mathematical morphological techniques using for removing the noise of region of the tumor on brain MRI image.Patel et al, [22] proposed to use K-means algorithm for identifying the tumor. Agarwal et al, [23] proposed to extract tumor from MRI images, where its method (TLS Algorithm) depended upon the size and shape of the tumor by applying segmentation by using global threshold based method that its value changing during the process till the end and designing the level set speed function for estimate its value accurately. They applied their algorithm by initializing the level set inside the MRI image then looking for the threshold, after that calculating the speed function then growing the level function and updating the threshold every time and then repeat the process by reinitializing the level set till finishing the process on the whole process. Their results showed the high performance of the algorithm and its efficiency.

\section{PROPOSED METHOD}

We have three algorithms (1), (2), and (3) were proposed for segmenting MRI images. We implemented MRI images as an input of medical image. We used median for removing noises from brain MRI images. K-means clustering was applied to segment based on level set.

\subsection{Image Acquisition}

For determining the diagnosis, in this process, it is based on hardware which is an imaging modality that is using for capturing the entire pathology organ and detecting it. So, they are based on the interaction with the patient's body. And after that software mission is based on formatting and converting the captured image to meaningful image of the diseased organ. And finally, the obtained image of the organ can be processed by using functions and algorithms for classification, enhancement, detection and segmentation that will be used to help in diagnosis process and taking an accurate decision for the status of the patient.

In this study, we used MRI imaging modality for brain imaging. We used several brain MRI images on our study. And we know that brain MRI image contains noise so we applied a preprocessing first before applying segmentation process to be able to obtain an accurate result. We have three steps with different algorithm. We explain three steps as following: pre-processing step for enhancing the image to be ready for segmentation, clustering step, and then apply level set method.

\subsection{Preprocessing}

In this step, we applied a noise filter on brain MRI image to reduce or remove the noise. MRI images most contain on noise so, when using these images for another processes for example segmentation, leading that we don't have an accurate results. Removing noise is a difficult task from MRI images.

There are different methods for reducing noise from medical images. Using image filtering for noise removing, contrast sharpening, contour highlightening, edge detection, and so on. In this study, we used one of the most important methods for reducing the noise. We used a non-linear filter, Median filter [24]. Median filter is one of the most important non-linear filtering for removing or reducing the noise on the images. It's an efficient method that can preserve the edge features. It's based upon on pixels with the principle of its neighborhood by moving pixel by pixel through the image until covering the whole image. It first sorts the pixel values and for odd masks and then replacing the target pixel by the median value after sorting. And numerous of studies confirm that median filter is better than linear filtering for removing the noise on the processed image [17].

In this step, Median filter [25] is working by sorting the values of an area on the brain MRI image in ascending order and then mixing these values by an odd mask with fixed size $3 \times 3$, 5X5, and 7 X7 respectively. For example, if we have the Figure 1.

\begin{tabular}{|c|c|c|}
\hline 40 & 20 & 66 \\
\hline 13 & 58 & 10 \\
\hline 86 & 15 & 150 \\
\hline
\end{tabular}

Figure 1. Block diagram for using Median filter 3X3 mask. 
Here, using Median filter and apply an odd 3X3 window on the image, it sorts the original image values on the full $3 \mathrm{X} 3$ window:

\title{
$10,13,15,20, \underline{40}, 58,66,86,150$
}

Median value is 40

Similary, using Median filter by same way and apply an odd 5X5 and an odd 7X7 window on the image and it sorts the original image values first on the full 5X5 and 7X7 window. We implemented our experiments by using MATLAB programming language. We used MATLAB R2017a. And here, the median filter algorithm could be summarized as follows:

\author{
Algorithm 1: Median filter algorithm \\ Input: brain MRI image \\ Output: filtered brain MRI image by median filter \\ 1- Read the brain MRI image. \\ 2- Dividing the image into blocks of size $3 \mathrm{X} 3$. \\ 3 - Sort the values of pixels in ascending order. \\ 4- Choose the middle value. \\ 5- Change the target pixel with middle value. \\ 6- Repeat from 2 to 5 until the process covered the whole image. \\ 7- End.
}

\subsection{Clustering algorithm}

Clustering is a technique that helping for understanding the structure of any image or an object. It is using for detecting, analyzing, and dividing the objects into parts that are called clusters and each cluster has same significant similarities. And these clusters have become a meaningful groups that can be used for image clustering, pattern recognition, data mining, bioinformatics, and data analysis [26, 27].

\subsubsection{K-means Clustering Algorithm}

$\mathrm{K}$-means algorithm is one of the most important unsupervised techniques [28, 16]. It is an efficient algorithm and was first mentioned by MacQueen 1967. It is used for clustering the object into different clusters with similarities characteristics. Its technique based upon the distance between these clusters, and their centers and purposes to minimize the sum squared error in the equation (1).

K-means algorithm classifies the data set from the vector space A into k clusters for minimizing the sum squared error $\mathrm{Z}$ in equation (1).

$$
\begin{aligned}
& \mathrm{A}\left(a_{1}, a_{2}, a_{3}, \ldots \ldots ., a_{n}\right) \text { where } \mathrm{n}=1,2,3 \ldots \mathrm{k} \\
& Z=\sum_{m=1}^{k} \sum_{t=1}^{n}\left\|a_{t}^{(m)}-C_{m}\right\|^{2}
\end{aligned}
$$

where

$$
C_{m}=\frac{1}{N_{m}} \sum_{a \in a_{t}} A
$$

$C_{m}$ is the clustering center, $\mathrm{m}=1,2,3 \ldots \mathrm{K}, N_{m}$ is the number of data set of the $m^{\text {th }}$ cluster $a_{m}$. And here, K-means algorithm could be summarized as follows:

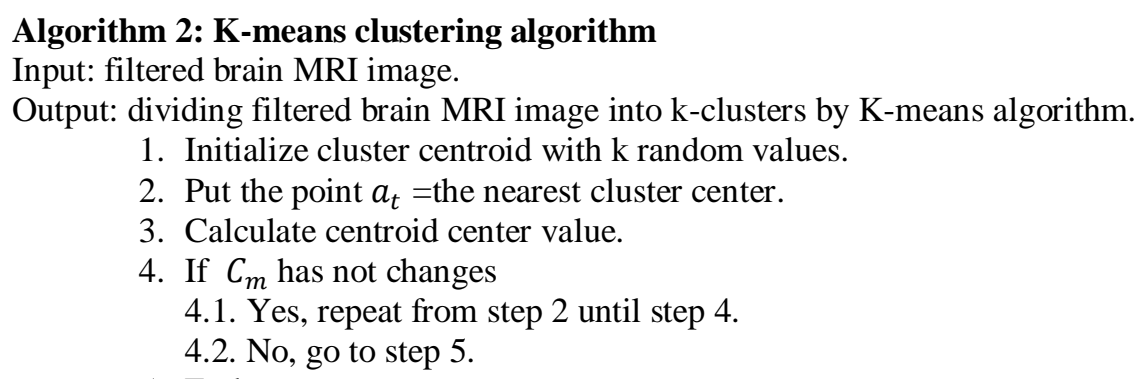

2. Put the point $a_{t}=$ the nearest cluster center.

3. Calculate centroid center value.

4. If $C_{m}$ has not changes

4.1. Yes, repeat from step 2 until step 4.

4.2. No, go to step 5 .

5. End. 


\subsection{Segmentation with level set method}

It's an implicit and important mathematical method which using for detecting shapes and objects which change topologically over time depending upon the theory of the curve and surface evolution. It was first mentioned by Osher and Sethian in 1988 [18]. Its consisting from levels, the surface or contours is considered as Zero level set and usually called a level set function. It defines the issue in one higher dimension. There are two types of level set formulations which include Time dependent level set formulation and Stationary level set formulation.

In image processing, some studies aimed for segmentation by using partial differential equations or calculus of variation for converting the continuous image into discrete image to be processed with level set method. It has an ability of implementing curves and surfaces on a fixed Cartesian grid without having to parameterize these objects on its task processing [29]. But, its algorithm is slow and does not implicitly preserve the level set function as a distance function.

Let $I=(m, n)$ is brain MRI image, wherem $\in\left[1, X_{m}\right], n \in\left[1, X_{n}\right]$, and $X$ is pixels numbers of the processed image. $I_{1}=(m, n)$ is a point of the brain MRI image and its changing with time changing. $w(t)$ is a position over the time, and every point $\mathrm{m}(\mathrm{t})$ is on the highest surface in the following equation (3).

$$
\alpha(w(t), t)=0
$$

Here, this method depends upon a mathematical function of partial differential equation $(\operatorname{PDF}) \alpha(t, m, n)$ and the evaluation is calculating by active contour by tracking Zero level set $\mathrm{w}(\mathrm{t})$ [30].

Figure 2 will summarize on the following equation (4):

$$
w(t)=\left\{\begin{array}{cc}
\alpha(t, m, n)<0 \quad(m, n) \text { is outside } w(t) \\
\alpha(t, m, n)>0 \quad(m, n) \text { is inside } w(t) \\
\alpha(t, m, n)=0,(m, n) \text { is on the boundary of } w(t)
\end{array}\right.
$$

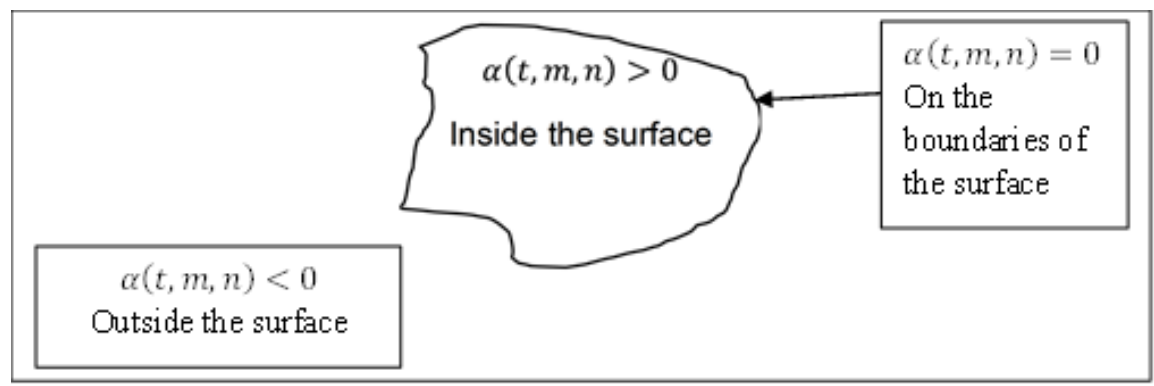

Figure 2. Level set function

The initial function $\alpha$ at $\mathrm{t}=0$ can be calculating by the following equations and we obtain

$$
\frac{\partial \alpha(w(t), t)}{\partial \alpha}=0
$$

By applying Chain rule [31]:

$$
\begin{aligned}
& \frac{\partial \alpha}{\partial w(t) \alpha} \cdot \frac{\partial w(t)}{\partial t}+\frac{\partial \alpha}{t} \cdot \frac{t}{t}=0 \\
& \frac{\partial \alpha}{\partial w(t)} m t+\alpha t=0
\end{aligned}
$$

By the following equation (8), we determine the function $\alpha$.

$$
\left\{\begin{array}{c}
\frac{\partial \alpha}{\partial t}+Z|\nabla \alpha|=0 \\
\alpha(0, m, n)=\alpha_{0}(m, n)
\end{array}\right.
$$

$|\nabla \alpha|$ is the natural direction, $\alpha_{0}(m, n)$ is initial contour. 
And for segmentation and ending the process and obtain the optimal solution, $\mathrm{Z}$ should be regularized by applying equation (9) and if it's value around zero or means it's on the boundary you follow to apply the equation (10).

$$
F=\frac{1}{1+\left|\nabla\left(G * I_{1}\right)\right|^{2}}
$$

$G * I_{1}$ is used for obtaining the image gradient by convolute brain MRI image with Gaussian noise. And then the famous level set for segmentation is:

$$
\frac{\partial \alpha}{\partial \alpha}=|\nabla \alpha|\left(\operatorname{div}\left(\frac{\nabla \alpha}{|\nabla \alpha|}\right)\right)
$$

And here, level set method algorithm could be summarized as follows:

\section{Algorithm 3: level set method algorithm}

Input: output brain MRI image from algorithm 2.

Output: segmented brain MRI image.

1 - Reading first cluster by using loop.

2- Testing for being inside, outside, or on the boundary

2.1. if $\alpha(t, m, n)>0$ go inside

2.2. if $\alpha(t, m, n)<0$ go outside

2.3. if $\alpha(t, m, n)=0$ go the boundary

3- Calculating initial function

4- Determine $\alpha$ by equation 8 .

5- if $\mathrm{F}$ on the boundary go to equation 9 and10.

6- Repeat all steps until finishing all clusters.

7- End.

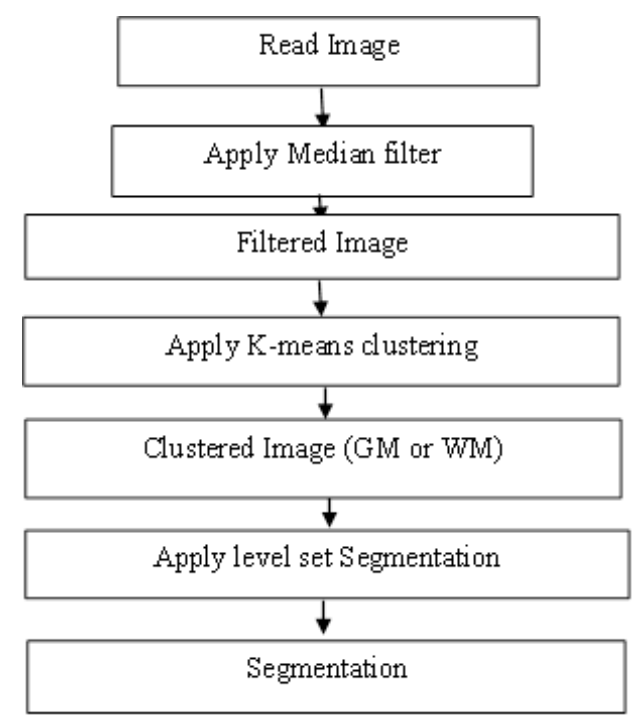

Figure 3. Flow chart of proposed method

\section{EXPERIMENT RESULTS}

MRI Images have noises and various diseases where every disease is different from the others in shapes, position, and sizes. By using a dataset of MRI brain images to prove the effeiciency of our proposed method and each step is presented and then comparing it with other previous used methods to evaluate which one is perfect. By applying Median filter on brain MRI image to remove noises that come out from the effect of Gaussian noise. We obtained on dataset from the following site (http://www.med.harvard.edu/AANLIB/home.html). By our method, it has the highest impact in 
computational processing with aconsiderable time consuming. The following Figure 4 represents original MRI images and its filtered results respectively

(a)
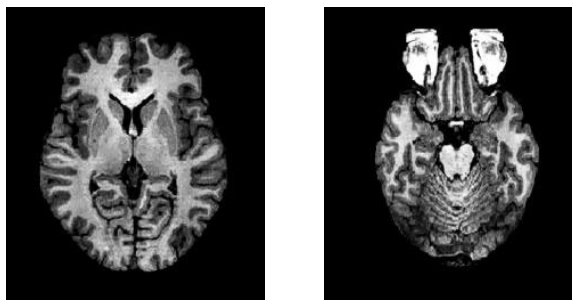

(b)
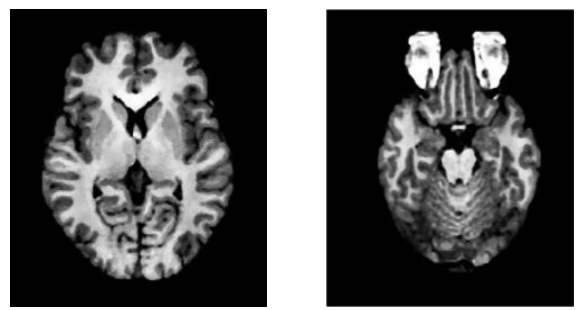
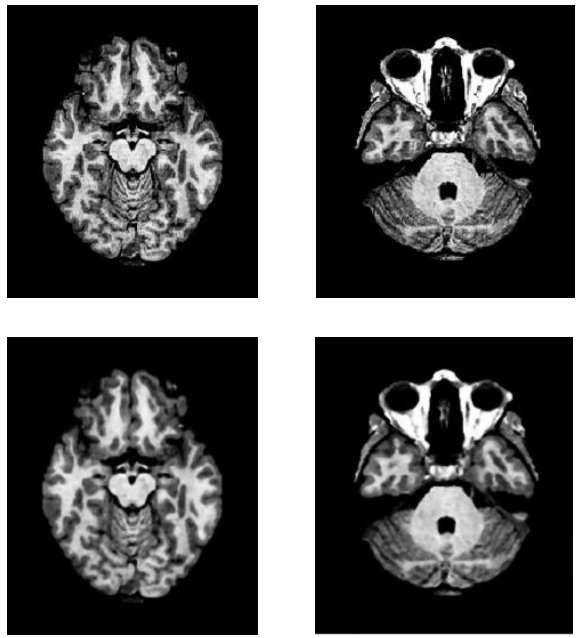

Figure 4. Row (a) represent an original MRI Images and row (b) shows its filtered results respectively

$\mathrm{K}$-means is applied for helping in the performance of the processing where was used for detecting the brain tissue into clusters in less time. This algorithm is used to detect the data into K numbers of alternately separated clusters. These separated clusters depend upon the corresponding center of each cluster where these center is calculated by the distance between the cluster center and data point. We proposed to filter MRI images first to obtain on the perfect results and achieving an accurate results on segmentation where K-means algorithm is sensitive and not familiar with the noise on the MRI images [28].

(a)

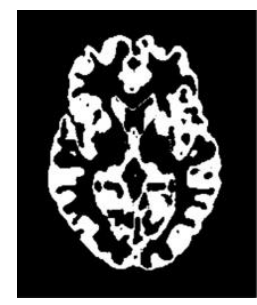

(b)

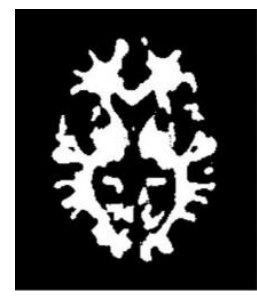

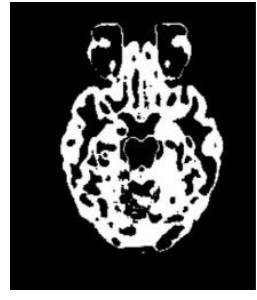



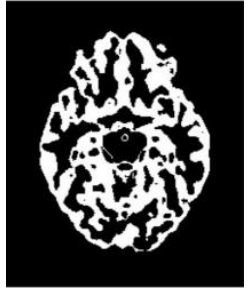

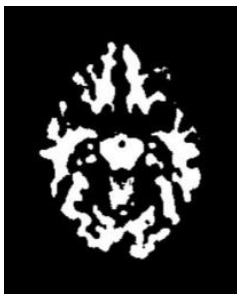

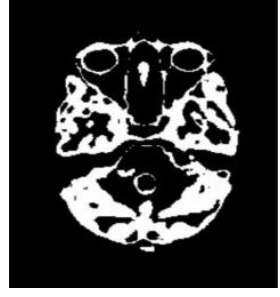

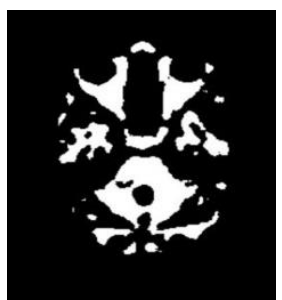

Figure 5. Row (a) shows clustered images for gray matters and row (b) shows clustered images for white matters of filtered images respectively

Table 1. The proposed method with a number of iterations and computational time in seconds for white and

\begin{tabular}{|c|c|c|c|c|c|c|c|}
\hline \multirow{6}{*}{ Time(s) } & \multirow{2}{*}{ Cases } & \multicolumn{3}{|c|}{ Gray matter cluseter image } & \multicolumn{3}{|c|}{ white matter cluseter image } \\
\hline & & Iter $=5$ & Iter $=10$ & Iter $=15$ & Iter $=5$ & Iter $=10$ & Iter $=15$ \\
\hline & Case1 & 1.841935 & 2.743089 & 3.100121 & 1.371474 & 2.102766 & 2.385045 \\
\hline & Case2 & 1.793302 & 2.953923 & 3.409964 & 2.022448 & 2.797612 & 3.856485 \\
\hline & Case3 & 1.739881 & 2.842646 & 3.337871 & 1.580557 & 2.179632 & 2.597748 \\
\hline & Case4 & 1.975437 & 2.708260 & 3.336627 & 2.094164 & 2.891269 & 3.462000 \\
\hline
\end{tabular}


In general, we proposed to achieve a perfect results of segmentation and clustering the dataset into clusters without time consuming. Our dataset is around 60 images and we clustered it into WM (white matter) and GM (gray matter). In our proposed method, we tended to use level set algorithm under number of iteration where level set algorithm helped to segment the brain tissue into GM and WM under dynamic variation limits and using a number of iteration which has an ability to affect on the quality of an image, in other algorithms of clustering in this step it may take a time (i.e. time increase) but, here K-means plays one of its important role in time consuming to be less. So, in our processing steps, we used a number of iteration of 5,10 , and the highest number of iteration we have used is 15 iterations. We evaluated the values of level set formulation as $\boldsymbol{\alpha}_{\mathbf{1}}=\mathbf{0 . 6}, \boldsymbol{\beta}_{\mathbf{1}}=\mathbf{0 . 4}, \boldsymbol{\lambda}_{\mathbf{1}}=\mathbf{0 . 2}$, and $\boldsymbol{\lambda}_{\mathbf{2}}=\mathbf{0} .3$ that has affect on the integral inside and outside the contour and $\boldsymbol{\lambda}_{1}$ and $\boldsymbol{\lambda}_{\mathbf{2}}$ shows the changes and affects that occurs inside and outside boundary during the process. Inspite of existing of weak boundaries, our proposed method has the ability of selecting and detecting the desired objects by using correntropy standard where we used in our experiments to change, decrease and increase of the values of $\boldsymbol{\alpha}_{\mathbf{1}}$ and $\boldsymbol{\beta}_{\mathbf{1}}$ to detect the accurate location of object boundaries in the Figure 6 and Figure 7.

(a)
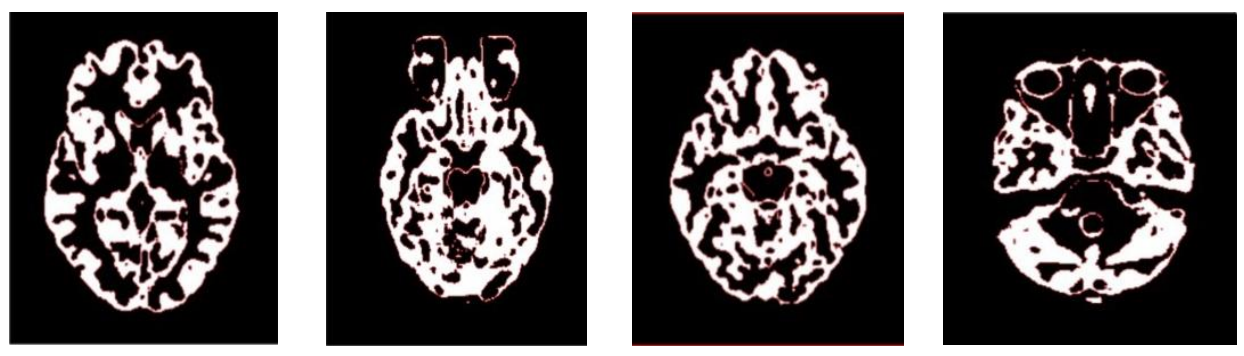

(b)
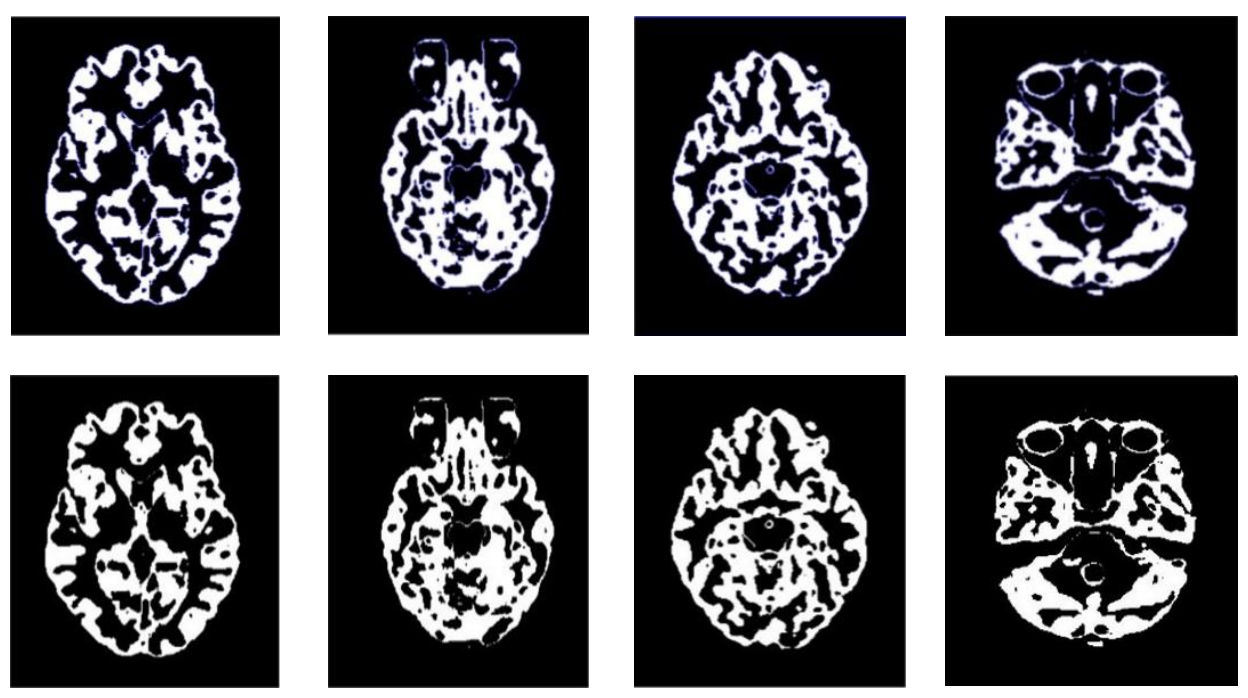

Figure 6. Brain MRI segmentation based on level set for clustered images by k-means for gray matters a, b, and $\mathrm{c}$ are segmentation after 5,10, and 15 iteration respectively with $\alpha_{1}=0.6, \beta_{1}=0.4, \lambda_{1}=0.2$, and $\lambda_{2}=$ 0.3 , where row (a) for starting segmentation, row (b) for final segmentation after 5,10, and 15, and row

(c) for extracting region of final segmentation

By comparing the proposed method by another algorithms used in previous studies applied on medical images [6] with a number of iteration and computational time(s) for white and gray matter in the Table 2 we can optimizing the performance of medical image segmentation. 
(a)
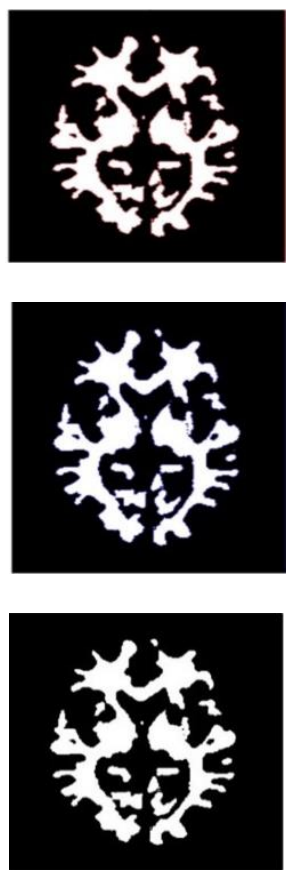
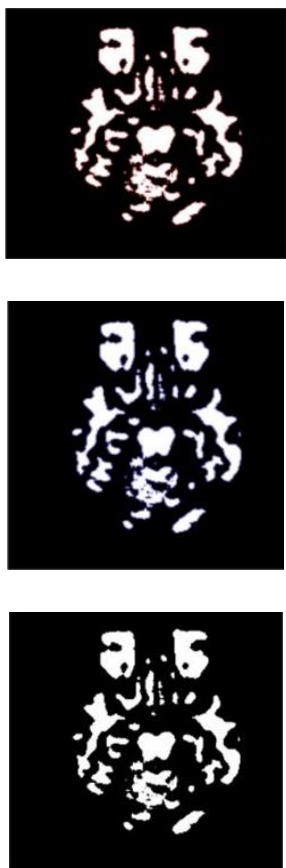
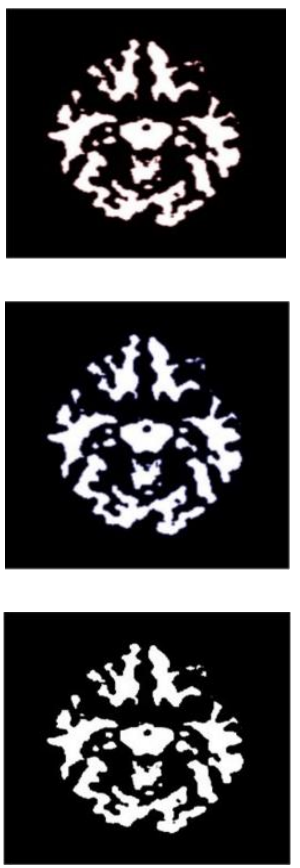
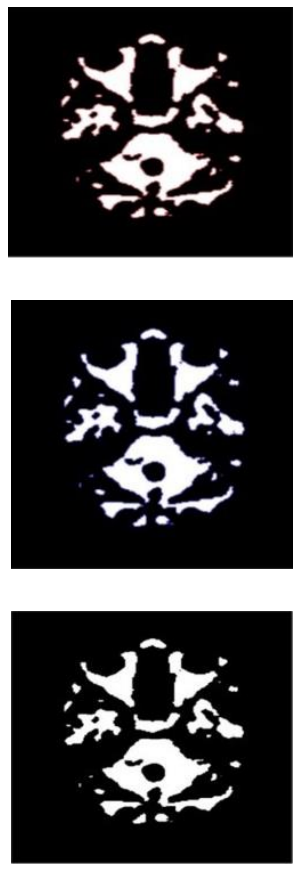

Figure 7. Brain MRI segmentation based on level set for clustered images by k-means for white matters a, b, and $\mathrm{c}$ are segmentation after 5,10, and 15 iteration respectively with $\alpha_{1}=0.6, \beta_{1}=0.4, \lambda_{1}=0.2$, and $\lambda_{2}=$ 0.3 , where row a: for starting segmentation, row $b$ : for final segmentation after 5,10 , and 15 , and row c: for extracting region of final segmentation

Table 2. Comparison between the proposed method (mfkmls) with another methods applied on medical images with a number of iteration and computational time (s) for white and gray matter

\begin{tabular}{|c|c|c|c|c|c|c|c|c|c|}
\hline Comparison & method & \multicolumn{4}{|c|}{ White matter cluster images } & \multicolumn{4}{|c|}{ gray matter cluster images } \\
\hline $\mathrm{LBF}$ & Time(s) & 31.51 & 5.20 & 21.79 & 4.30 & 31.51 & 5.20 & 21.79 & 4.30 \\
\hline LGDF & time(s) & 99.52 & 12.47 & 9.33 & 29.40 & 99.52 & 12.47 & 9.33 & 29.40 \\
\hline \multirow{2}{*}{ LCK } & iteration & 300 & 580 & 260 & 140 & 300 & 580 & 260 & 140 \\
\hline & time(s) & 78.87 & 21.16 & 43.73 & 6.01 & 78.87 & 21.16 & 43.73 & 6.01 \\
\hline
\end{tabular}

\section{CONCLUSION}

Filtering noise from around $60 \mathrm{MRI}$ images by using Median filter helped for removing the noise and enhancing the results of segmentation. There is no MRI images that empty from noise and K-means is sensitive to noise so filtering first was the best to be done, but K-means is a best way for managing the time of the processing. After comparison with other algorithms that were done for another medical images, our proposed method performance is perfect for segmentation without time consuming too much.

\section{ACKNOWLEDGMENT}

This work is supported by the Science and Technology Program of Shenzhen of China under Grant Nos. JCYJ20170818160208570 and JCYJ20170307160458368.

\section{REFERENCES}

[1] Wen P., et al., "Spatial Credibilistic Clustering Algorithm in Noise Image Segmentation," 2007 International Conference on Industrial Engineering and Engineering Management, Singapore, pp. 543-547, 2007.

[2] Dhawan A. P., "A review on biomedical image processing and future trends," Comput Methods Programs Biomed, vol. 31, pp. 141-83, 1990. 
[3] Umamaheswari J. and Radhamani G., "A fusion technique for medical image segmentation," 2012 Int Conf Devices, Circuits Syst ICDCS 2012. Coimbatore, India, pp. 653-657, 2012.

[4] Zhang H., et al., "Image segmentation evaluation: A survey of unsupervised methods," Comput Vis Image Underst., vol. 110, pp. 260-280, 2008.

[5] Peng B., et al., "Automatic image segmentation by dynamic region merging," IEEE Transactions on Image Processing, vol. 20, pp. 3592-3605, 2011.

[6] Jiang X. L., et al., "Robust level set image segmentation algorithm using local correntropy-based fuzzy c-means clustering with spatial constraints," Neurocomputing, vol. 207, pp. 22-35, 2016.

[7] Yqub I., et al., "Fuzzy segmentation of MRI cerebral tissue using level set algorithm," Int.J Innovative Research in Information Security, vol. 5, pp. 25-35, 2018.

[8] Küçükkülahli E., et al., "Brain MRI Segmentation based on Different Clustering Algorithms," Int J Comput Appl., vol. 155, pp. 37-40, 2016.

[9] Duraisamy M. and Jane F. M. M., "Cellular neural network based medical image segmentation using artificial bee colony algorithm," Proceeding IEEE Int Conf Green Comput Commun Electr Eng ICGCCEE 2014. Coimbatore, India, pp. 1-6, 2014.

[10] Logeswari T. and K. Mrcus, "An Enhanced Implementation of Brain Tumor Detection Using Segmentation Based on Soft Computing," 2010 International Conference on Signal Acquisition and Processing. Bangalore, vol. 2, pp. 243-247, 2010.

[11] Selvy P. T., et al., "Performance Analysis of Clustering Algorithms in Brain Tumor Detection of MR Images," Eur J Sci Res., vol. 62, pp. 321-330, 2011.

[12] Ahmadvand A. and D. M. Reza, "Brain MR Image Segmentation Methods and Applications," Omi J Radiol., vol. 02, pp. 3-5, 2014.

[13] Dunn J. C., "A fuzzy relative of the ISODATA process and its use in detecting compact well-separated clusters," $J$ Cybern, vol. 3, pp. 32-57, 1973.

[14] Kamble S. T. and Rathod M. R., "Brain Tumor Segmentation using K-Means Clustering Algorithm,” vol. 5, pp. 1521-1524, 2015.

[15] M. Ahmed and I. Saiful, "Implementation of Image Segmentation for Natural Images using Clustering Methods," Int J Emerg Technol Adv Eng., vol. 3, 2013.

[16] Liu J. and Guo L., "A new brain MRI image segmentation strategy based on K-means clustering and SVM," Proc 7th Int Conf Intell Human-Machine Syst Cybern IHMSC. Hangzhou, Nanjing, China, vol. 2, pp. 270-273, 2015.

[17] Arias C. E. and Donoho D. L., "Does median filtering truly preserve edges better than linear filtering?" Ann Stat., vol. 37, pp. 1172-1206, 2009.

[18] O. Stanley and Sethian J. A., "Fronts Propagating with Curvature- Dependent Speed: Algorithms Based on Hamilton-Jacobi Formulations," Instruments Exp Tech New York, vol. 26, pp. 178-179, 1988.

[19] Rastgarpour M. and Shanbehzadeh J., "The Problems, Applications and Growing Interest in Automatic Segmentation of Medical Images from the Year 2000 till 2011," Int J Comput Theory Eng., vol. 5, pp. 1-4, 2013.

[20] Abd-Ellah M. K., et al., "Design and implementation of a computer-aided diagnosis system for brain tumor classification," Proc 28th Int Conf Microelectron ICM. Beirut, Lebanon, pp. 73-76, 2016.

[21] Koley S. and Majumder A., "Brain MRI segmentation for tumor detection using cohesion based self merging algorithm," Commun Softw Networks (ICCSN), 2011 IEEE 3rd Int Conf. Xi'an, China, pp. 781-785, 2011.

[22] Patel P. M., et al., "Image segmentation using K-mean clustering for finding tumor in medical application," Int $J$ Comput Trends Technol., vol. 4, pp. 1239-1242, 2013.

[23] Agarwal V. P. and Kumar M., "Tumor Extraction by Level Set Method using Thershold Algorithm," Int J Comput Appl., vol. 94, pp. 975-8887, 2014.

[24] Bhateja V., et al., "A non-iterative adaptive median filter for image denoising," 2014 Int Conf Signal Process Integr Networks. Delhi, India, pp. 113-118, 2014.

[25] Kaur A., et al., "Performance evaluation of non-iterative adaptive median filter," Souvenir 2015 IEEE Int Adv Comput Conf IACC 2015. Bangal, pp. 1117-1121, 2015.

[26] Ding Y. and Fu X., "Kernel-based fuzzy c-means clustering algorithm based on genetic algorithm," Neurocomputing, vol. 188, pp. 233-238, 2016.

[27] Jain A. K., et al., "Statistical pattern recognition: A review," IEEE Trans Pattern Anal Mach Intell., vol. 22, pp. 4$37,2000$.

[28] Somasundaram K. and Genish T., "Binarization of MRI with Intensity Inhomogeneity using K- Means Clustering for Segmenting Hippocampus," Int J Multimed Its Appl., vol. 5, pp. 11-9, 2013.

[29] Vineetha G. R. and Darshan G., "Level Set Method for Image Segmentation: A Survey," IOSR J Comput Eng., vol. 8, pp. 74-78, 2013.

[30] Friedrich T., et al., "The compact genetic algorithm is efficient under extreme Gaussian noise," IEEE Trans Evol Comput., vol. 21, pp. 477-490, 2017.

[31] A. R. Ben, et al., "GPU-based Segmentation of Dental X-ray Images using Active Contours without Edges," 15th International Conference on Intelligent Systems Design and Applications. Marrakesh, Morocco, pp. 505-510, 2015. 